\title{
Influence of Initial Energy Selection in Leach Protocol based Wireless Sensor Networks
}

\author{
Shinu Kansal \\ Department of Electronics \& \\ Communication Engineering \\ Punjabi University Patiala, \\ 147002, Punjab, India
}

\author{
Manjit Singh Bhamrah \\ Department of Electronics \& \\ Communication Engineering \\ Punjabi University Patiala, \\ 147002, Punjab, India
}

\author{
Gautam Kaushal \\ Department of Electronics \& \\ Communication Engineering Punjabi \\ University Patiala, 147002, Punjab, \\ India
}

\begin{abstract}
The paper investigates the change of initial energy in leach protocol to prolong the network lifetime in order to achieve network reliability and scalability. The values of initial energy taken are $0.5,1,2,3$ joule. For the case, simulation results show that nodes start decaying after $5100^{\text {th }}$ rounds. Thus nodes are surviving longer for higher energy cases taken. The work recommends adequate energy use to increase network lifetime of Wireless Sensor Networks.
\end{abstract}

Key words-Wireless Sensor Network, Leach Protocol, Clustering, Network Lifetime

\section{INTRODUCTION}

In Recent years, a wireless sensor network has been become the furthermost exciting networking technologies to forward the sensed collected data to the base station with restricted energy capacity. Wireless sensors are micro electro-mechanical system devices

that has been sensing, processing and communication capabilities. A wireless sensor network system usually includes sensor nodes, base station and management node [1]. Wireless sensor network (WSN) has been extensively applied as a fundamental technology of various applications. In these applications like E-health, military, home, traffic uses a large number of sensor nodes which have restricted resources (e.g., computing, energy) [2]. A primary design goal for wireless sensor networks has been to use the energy efficiently. The cluster-based routing algorithms group nearby sensors together to form a number of clusters. Each cluster has one representative sensor called Cluster Head $(\mathrm{CH})$. $\mathrm{CHs}$ are required to collect data from other sensors in their cluster and send the aggregated data to the base station. Generally, all sensors do not interact with the base station, hence, more energy is saved and the network lifetime is increased $[3,4]$. The basic idea of clustering routing has been to use the information a aggregation mechanism in the cluster head to reduce the amount of data transmission, thereby, reduce the energy dissipation in communication and in turn achieve the purpose of saving energy of the sensor nodes.

In the clustering routing algorithms for wireless networks, LEACH (low-energy adaptive clustering hierarchy) show significant performance improvements in terms of network lifetime and throughput [3,5]. LEACH has been first proposed by Wendi B. Heinzelman. This protocol provides a conception of round. LEACH protocol runs with many rounds. Each round contains two phases: cluster setup phase and steady phase. In the setup phase, each node decides whether or not to become a cluster head for current round. The selection depends on decision made by the node by choosing a random number between 0 and 1 . If the number is less than the threshold $T(n)$ [6], the node becomes a cluster head for the current round. The threshold is set as:

$T(n)=\left\{\begin{array}{lrr}\frac{p}{1-p \cdot\left(r \times \bmod \frac{1}{p}\right)}, & n \in G \\ 0, & \text { otherwise } & \ldots \ldots . .(1)\end{array}\right.$

Where $p$ is the probability of the node being selected as a cluster-head node, $r$ is the number of rounds passed, and $G$ is the set of nodes that have not been cluster-heads in the last $1 / p$ rounds, mod denotes modulo operator nodes that are cluster heads in round $r$ shall not be selected in the next $1 / p$ rounds. Once the cluster-head is selected, all nodes join the corresponding cluster according to the broadcast signal intensity of the cluster-head node. Then, the cluster set-up phase of this round is completed. When the cluster-head assigns time slots for members using TDMA mode, the network will enter the steady phase. The steady phase is divided into frame, where nodes send their data to the cluster head at most once per frame during their allocated transmission slot [14].

LEACH has many advantages. It has been reduces the amount of information sent to the sink node. It based on TDMA/CDMA MAC layer mechanism to reduce the conflict between clusters. Since data collection is concentrated and cyclical, LEACH is ideal for applications that require continuous monitoring system [13].

Already there has been a lot of research work based upon LEACH to make this protocol more reasonable and efficient, such as LEACH-C, LEACH with MTE routing , TL-LEACH , TB-LEACH, LEACH with MECH routing, DAIC-LEACH, LEACH-DC, LEACH-MF, LEACH-R, E-LEACH, EN-LEACH, FZ-LEACH, V-LEACH [6-18], etc. but there is further scope to investigate Leach protocol by varying simulation parameters like network size, number of nodes, probability of cluster head, and size of data packet..

It is also seen that LEACH protocol is further improved by varying initial energy of nodes. This work presents simulation results, for a set of initial energy of node: 0.5 , 1,2 , and $3 \mathrm{~J}$. 


\section{MODEL DESCRIPTION}

The conventional radio model for LEACH of first order is considered [14].

In this model, energy consumption of sending $l$ bit is expressed as following equation (2).

$$
\begin{aligned}
& E_{T X}(l, d)=\left\{E_{\text {elect }} \times l+\varepsilon_{f S} \times l \times d^{2} \quad d<d_{0}\right. \\
& E_{\text {elect }} \times l+\varepsilon_{\text {amp }} \times l \times d^{4} d \geq d_{0} \\
& \text {......... (2) } \\
& d_{0}=\sqrt{\frac{\varepsilon_{f s}}{\varepsilon_{a m p}}} .
\end{aligned}
$$

The distance threshold value is expressed as equation (3), $\varepsilon$ is amplification factor of signal amplifier, and elect $E$ is energy consumption of transmitting and receiving circuit. In this model, it is thought that they are equal, $r$ is decided by radio channel model, when the transmission distance is less than distance threshold, free space model is used, $r=$ 2 , if more than distance threshold, multipath attenuation model is used, $r=4$.

Energy consumption of receiving $l$ is expressed as equation (4).

$$
E_{R X}(l, d)=E_{\text {elect }} \times l
$$

\section{Table 1.Simulation Parameter}

\begin{tabular}{|l|l|}
\hline Parameter & Value \\
\hline Number of Nodes & 100 \\
\hline Network Grid & $100 \times 100 \mathrm{~m}^{2}$ \\
\hline$\varepsilon_{\mathrm{fs}}$ & $10 \mathrm{pJ} / \mathrm{bit} / \mathrm{m}^{2}$ \\
\hline$\varepsilon_{\text {amp }}$ & $0.0013 \mathrm{pJ} / \mathrm{bit} / \mathrm{m}^{4}$ \\
\hline $\mathrm{E}_{\text {elect }}$ & $50 \mathrm{~nJ} / \mathrm{bits}$ \\
\hline $\begin{array}{l}\text { Maximum no. of } \\
\text { rounds }\end{array}$ & 8000 \\
\hline Size of data packet & 4000 bytes \\
\hline $\begin{array}{l}\text { Probability of cluster } \\
\text { Head }\end{array}$ & $10 \%$ \\
\hline Initial Energy & $0.5,1,2,3$ Joules \\
\hline
\end{tabular}

\section{RESULTS \& DISCUSSION}

The figure 1. Shows that as on increase the initial energy, the node becomes start decaying after more rounds elapse. When initial energy (joules per node) is $0.5,1,2,3$ then nodes starts dying after $800^{\text {th }}, 1600^{\text {th }}, 3100^{\text {th }}, 5100^{\text {th }}$ rounds respectively. If nodes have less initial energy, it consumes energy to become cluster head more and nodes die quickly. As increasing initial energy then nodes consuming less energy to become cluster head and doesn't die quickly. So nodes remain alive for more time $\&$ hence increases the network lifetime of system.

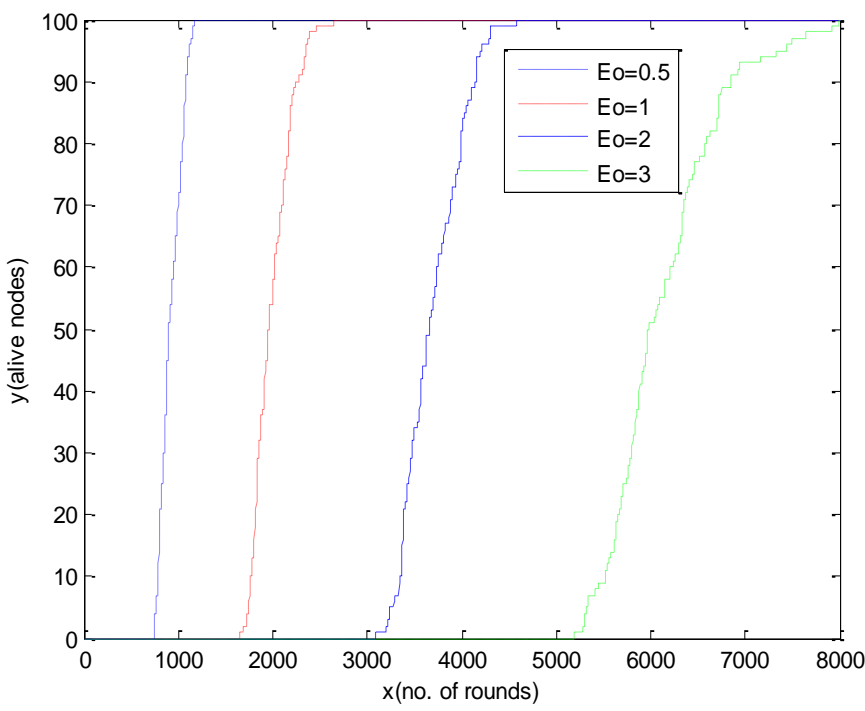

Figure 1. Alive nodes versus number of rounds tell the network lifetime of system for the specific initial energies (i.e. $\mathbf{E}_{0}$ in joules) cases: a) 0.5 b) 1 c) 2 d) 3

Table 2. Network Lifetime of Wireless Sensor Networks for various Initial energies (i.e. $E_{0}$ in joules) cases: a) 0.5 b) 1 c) 2 d) 3

\begin{tabular}{|c|c|}
\hline $\begin{array}{c}\text { Initial Energy } \\
\text { (Joules/nodes) }\end{array}$ & $\begin{array}{c}\text { Rounds when nodes } \\
\text { start dying }\end{array}$ \\
\hline 0.5 & $800^{\text {th }}$ \\
\hline 1 & $1600^{\text {th }}$ \\
\hline 2 & $3100^{\text {th }}$ \\
\hline 3 & $5100^{\text {th }}$ \\
\hline
\end{tabular}




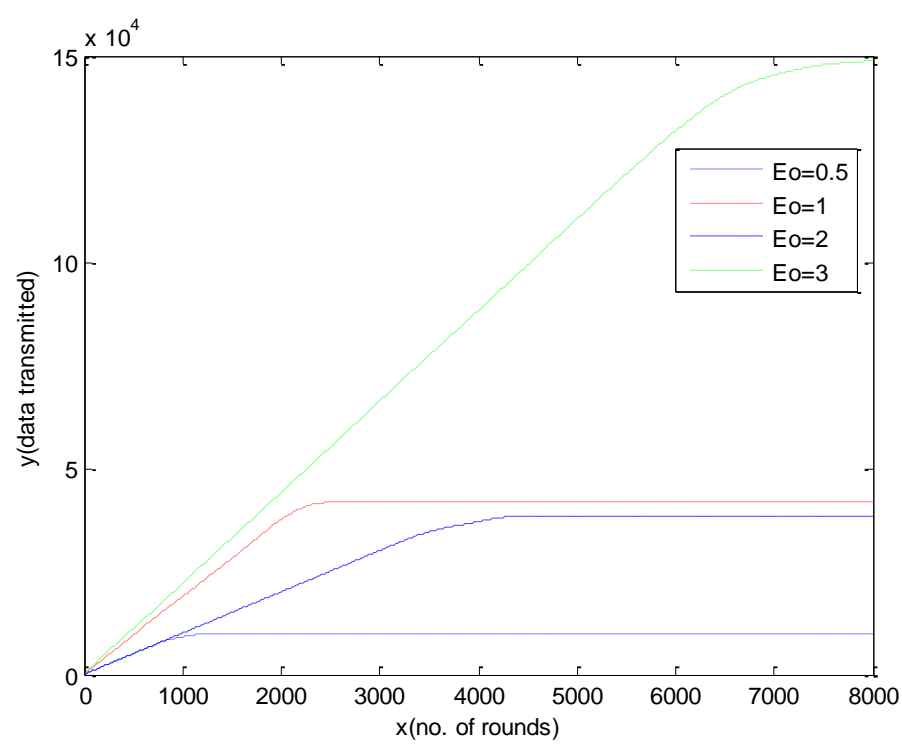

Figure 2. Data transmitted to base station versus number of rounds for the specific initial energies (i.e. $\mathrm{E}_{0}$ in joules) cases: a) 0.5 b) 1 c) 2 d) 3

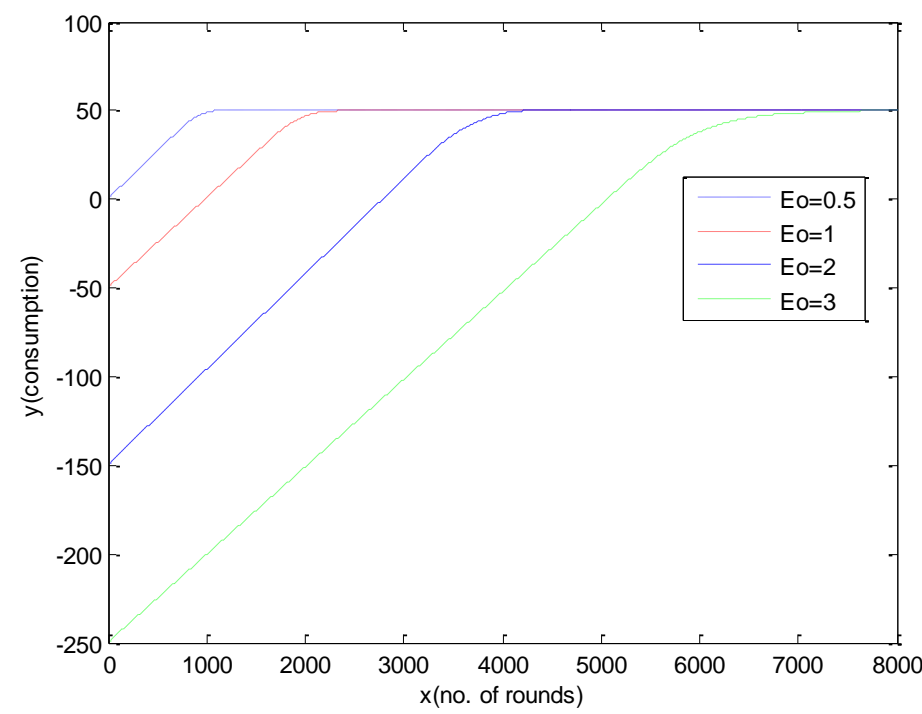

Figure 3. Energy Consumed by nodes while passing through number of rounds with taken initial energy (i.e. $E_{0}$ in joules) cases: a) 0.5 b) 1 c) 2 d) 3 


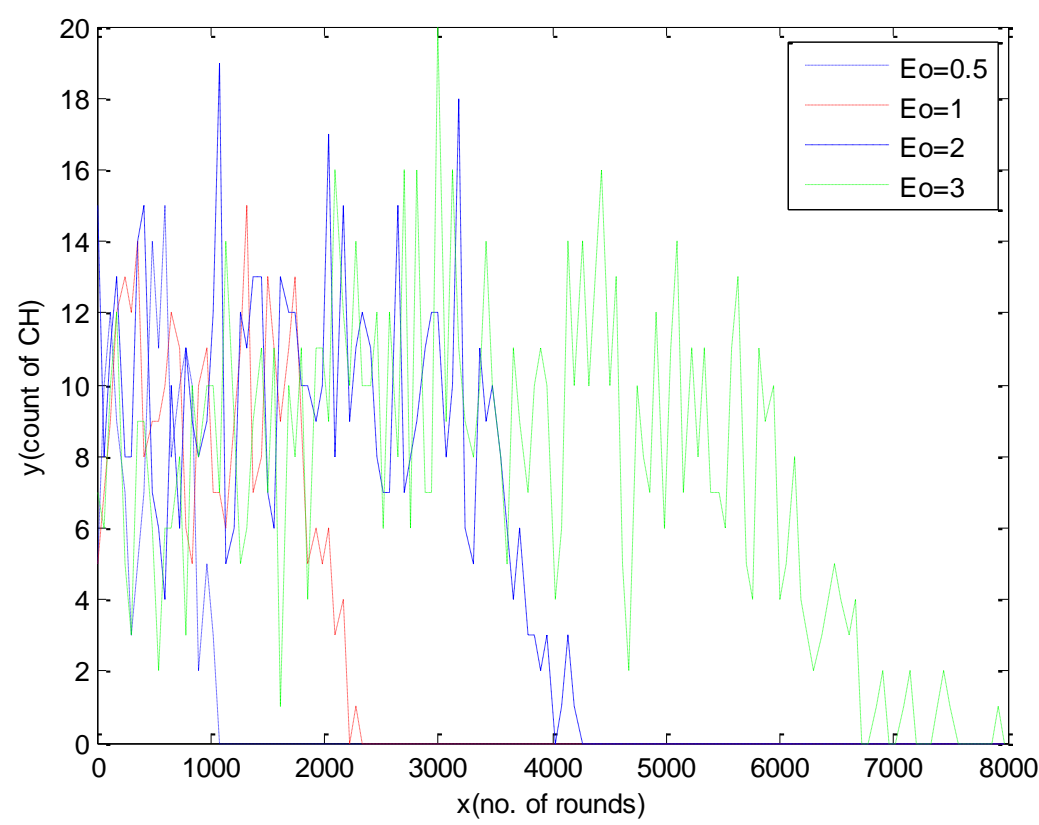

Figure 4 Counting number of CH's versus number of rounds during different initial energy (i.e. $\mathrm{E}_{0}$ in joules) cases: a) 0.5 b) 1 c) 2 d) 3

As shown in figure 2. With increase the initial energy the data transmitted to BS increase. While transmitting data to base station, nodes consumes an amount of power. When the value of initial energy of node set is $3 \mathrm{~J}$ then data transmitted to BS is 14 times more than $0.5 \mathrm{~J}$ value.

As figure 3. shows that with increase the initial energy the time duration of energy of node increases. A node spends energy in first order radio model according to data transmitted to BS.Concered graph shows that energy consumption increasing linearly with rounds when nodes selected as CH's and after that its energy exhaust then it becomes constant.

The figure 4. depicts the counting of nodes that become cluster head after elapsing number of rounds during each initial energy cases.

From the [17] Zhi-feng Duan, Fan Guo, Ming-Xing Deng and $\mathrm{Min} \mathrm{Yu}$, set the initial energy of $0.25,0.5,1 \mathrm{~J}$ throughout the $100 \times 100$ network area \& simulation results shows that nodes dies at $854^{\text {th }}, 1705^{\text {th }}, 3257^{\text {th }}$ respectively.

During investigation, the initial energy values $0.5,1,2,3 \mathrm{~J}$ throughout the $100 \times 100$ network area are taken $\&$ results show that nodes start decaying at $800^{\text {th }} 1600^{\text {th }}, 3100^{\text {th }}$, $5100^{\text {th }}$ rounds respectively. Investigation result shows that with small increase in initial energy values it increases the network lifetime of systems.

\section{CONCLUSIONS}

In this paper, study a parameter of LEACH protocol in first order radio model \& explored the life time under the variation of initial energy of nodes. The investigations suggested that on increasing the initial energy of nodes within same network grid the network lifetime of network increases because of nodes consuming less energy and surviving at $5100^{\text {th }}$ rounds \& after that few nodes start decaying.
In future, scope of research will be on improving the method of the Cluster head selection among the nodes to balance the energy $\&$ to achieve longer lifetime of wireless sensor networks.

\section{REFERENCES}

[1] Ningbo Wang, Hao Zhu, "An energy efficient algorithm based on LEACH protocol," International Conference on Computer Science \& Electronics Engineering, 2012.

[2] Do Sung Kim, Hyun Soo Cha, Seungwha Yoo, "Improve Far-Zone LEACH protocol foe energy conserving," IEEE, 2012.

[3] Yun li, Nan Yu, Weiyi Zhang, "Enhancing the performance of LEACH protocol in Wireless Sensor Networks," IEEE Infocom, 2011.

[4] W. Heinzelman, A. Chandrakasan, and H. Balakrishnan, "Energy efficient communication protocol for wireless Microsensor networks," in: Proceedings of the Hawaii International Conference on System Sciences, Hawaii, January, 2000.

[5] Z. Zhang and X. Zhang., "Research of Improved Clustering Routing Algorithm Based on Load Balance in Wireless Sensor Networks", IET International Communication Conference on Wireless Mobile and Computing, 2009, 661-664.

[6] W. Heinzelman, A. Chandrakasan, and H. Balakrishnan, "An application-specific protocol architecture for wireless Microsensor networks," IEEE Transactions on Wireless Communications, Vol. 1, No.4, pp. 660-670, Oct. 2002.

[7] Jichuan Zhao, A. Erdogan and T. Arslan, "A novel application specific network protocol for wireless sensor networks," in: IEEE International Symposium on Circuits and Systems, 2005, pp.5894-5897. 
[8] V. Loscri, G. Morabito, and S. Marano, "A two-level hierarchy for low energy adaptive clustering hierarchy (TL-LEACH)," in: Proceedings of IEEE 62nd Vehicular Technology Conference, 2005, pp.1809-1813.

[9] Hu Junping, Jin Yuhui, Dou Liang, "A Time-based Cluster-Head Selection Algorithm for LEACH," School of Information Engineering, Wuhan University of Technology, Wuhan China, IEEE, 2008.

[10] Ruay-Shiung Chang and Chia-Jou Kuo, "An energy efficient routing mechanism for wireless sensor networks," in: Proceedings of IEEE International Conference on Advanced Information Networking and Application, 2006.

[11] Navin Gautam, Jae-Young Pyun, "Distance Aware Intelligent Clustering Protocol for Wireless Sensor Networks," Journal of Communications and Networks, Vol. 12, NO. 2, April 2010.

[12] LIU Zhi-jun, LI La-yuan, "Based on Energy Balance LEACH-DC Protocol Design," Department of Computer and Information Engineering Wuhan Bioengineering Institute Wuhan, China, IEEE, 2011.

[13] Jian-Feng-Yan, Yuan-Liu Liu, "Improved LEACH Routing Protocol For Large Scale Wireless Sensor Networks Routing," School of Computer Science \&
Technology Soochow University Soochow 215006, China, IEEE, 2011.

[14] Jia Xu,Ning Jin, Xizhong Lou,Ting Peng,Qian Zhou,Yanmin Chen, "Improvement of LEACH protocol for WSN ," 9th International Conference on Fuzzy Systems and Knowledge Discovery, 2012.

[15] Suyog Pawar, Prabha Kasliwal, "Design and Evaluation of En-LEACH Routing Protocol for Wireless Sensor Network," International Conference on Cyber-Enabled Distributed Computing and Knowledge Discover, 2012.

[16] Fan Xianging, Song Yulin, "Improvement on LEACH Protocol of Wireless Sensor Network," International Conference on Sensor Technologies and Applications, 2007.

[17] Zhi-feng Duan, Fan Guo, Ming-Xing Deng and Min $\mathrm{Yu}$, " Shortest Path Routing Protocol for Multi-layer Mobile Wireless Sensor Networks", International Conference on Networks Security, Wireless Communications and Trusted Computing, 2009.

[18] Asha Ahlawat, Vineeta Malik, "An extended vicecluster selection approach to improve V LEACH protocol in WSN," LEACH Third International Conference on Advanced Computing Communication Technologies, 2013. 